\title{
Satisfacción de aspiraciones profesionales de jóvenes universitarios cubanos
}

Yisell Aguilar Pino ${ }^{1}$ Deborah Rodríguez Ravelo² Keyla Rosa Estévez García ${ }^{3}$

\begin{abstract}
Resumen:
El presente artículo se basa en un proyecto de investigación que se realiza en el Centro de Estudios sobre la Juventud: Satisfacción de las aspiraciones profesionales de jóvenes universitarios cubanos en el período 2020-2021. Se aborda una caracterización de las principales variables a investigar: satisfacción y aspiración profesional a partir de estudios nacionales e internacionales. Se explican los modelos motivacionales que sirvieron de base a los estudios sobre satisfacción y los factores de la relación estudiante-Universidad que influyen en dicho proceso. Además, se destaca la importancia que tiene el problema de investigación para la ciencia actual a través de la revisión bibliográfica. Se describen los beneficios que aporta el estudio de la satisfacción de aspiraciones profesionales para el bienestar estudiantil y el desarrollo de la institución educativa. Es inconcluyente la información sobre el nivel de satisfacción de aspiraciones profesionales debido a que el estudio se encuentra en proceso de aplicación, pero se plantean reflexiones a partir de resultados de investigaciones que se han realizado en el país.
\end{abstract}

\author{
Palabras claves: \\ Satisfacción. Aspiraciones. Jóvenes universitarios. Educación superior.
}

\section{Satisfação das aspirações profissionais dos jovens estudantes universitários cubanos}

Resumo: Este artigo é baseado em um projeto de pesquisa realizado no Centro de Estudos da Juventude: Satisfação das aspirações profissionais dos jovens universitários cubanos no período de 2020-2021. Ela aborda uma caracterização das principais variáveis a serem investigadas: satisfação e aspiração profissional a partir de estudos nacionais e internacionais. Os modelos motivacionais que serviram de base

\footnotetext{
1 Licenciada en Psicología (2019), Universidad de La Habana, Facultad de Psicología. Especialista en Investigaciones Sociales del Centro de Estudios Sobre la Juventud en el Departamento de Política y Sociedad. E-mail: yisellaguilarpino@gmail.com. ORCID iD: http://orcid.org/0000-0001-7037-2959.

2 Licenciada en Psicología (2012), Universidad de Ciencias Médicas de La Habana, Facultad “Manuel Fajardo". Especialista en Investigaciones Sociales del Centro de Estudios Sobre la Juventud en el Departamento de Política y Sociedad. E-mail: debi1902@nauta.cu. ORCID iD: http://orcid.org/0000-0001-8538-3298.

3 Doctora en Ciencias Pedagógicas por el ICCP (2008), Investigadora Auxiliar del Centro de Estudios sobre Juventud y se desempeña como Jefa del Departamento de Investigación Política y Sociedad. E-mail: jinvsociop@cej.ujc.cu. ORCID iD: http:// orcid.org/0000-0003-1407-2542.
} 
para os estudos sobre satisfação e os fatores da relação aluno-universidade que influenciam este processo são explicados. Além disso, a importância do problema da pesquisa para a ciência atual é destacada através da revisão bibliográfica. Os benefícios de estudar a satisfação das aspirações de carreira para o bem-estar dos estudantes e o desenvolvimento da instituição educacional são descritos. As informações sobre o nível de satisfação das aspirações de carreira são inconclusivas porque o estudo está em processo de implementação, mas as reflexões são levantadas a partir de resultados de pesquisa que foram conduzidos no país.

Palavras-chave: Satisfação. Aspirações. Jovens estudantes universitários. Ensino superior.

\section{Satisfaction of professional aspirations of young Cuban university students}

Abstract: This article is based on a research project carried out at the Centro de Estudios Sobre la Juventud: Satisfaction of the professional aspirations of young Cuban university students in the period 2020-2021. It presents a characterization of the main variables to be investigated: satisfaction and professional aspiration based on national and international studies. The motivational models that served as a basis for the studies on satisfaction and the factors of the student-university relationship that influence this process are explained. In addition, the importance of the research problem for current science is highlighted through the bibliographic review. The benefits of studying the satisfaction of professional aspirations for student welfare and the development of the educational institution are described. The information on the level of satisfaction of professional aspirations is inconclusive due to the fact that the study is in the process of implementation, but reflections are made based on the results of research that has been carried out in the country.

Keywords: Satisfaction. Aspirations. Young university students. Higher education.

\section{Introducción}

Resultados de investigaciones internacionales sobre la satisfacción estudiantil han demostrado los beneficios que aporta este fenómeno en los jóvenes estudiantes y las ganancias que les otorga a las instituciones educativas. La mayoría de los estudios de satisfacción se dedican a explorar la calidad de la formación educativa para llevar a cabo perfeccionamientos que garanticen la permanencia estudiantil, la lealtad y el prestigio de la Universidad. Sin embargo, pocas son las investigaciones que han abordado sobre la satisfacción de aspiraciones profesionales.

El interés por estudiar este fenómeno surge por los resultados obtenidos de Encuestas Nacionales de Juventud donde los jóvenes cubanos responden que aspiran a tener estudios superiores. Ante esta respuesta surge la inquietud de si las aspiraciones profesionales de los jóvenes se satisfacen. Es por ello que nos cuestionamos cómo la Universidad satisface estas aspiraciones, en dónde encuentran su satisfacción, así como qué elementos influyen en esta. La investigación se centra en comprender la relación estudiante-universidad, entendiéndose a nivel internacional como una relación esencialmente comercial, mientras que en Cuba se comprende como un servicio formativo del estudiante sin carácter comercial donde éste no es un sujeto pasivo, sino que puede ser capaz de investigar a partir de sus propios intereses.

El presente artículo pretende esclarecer las definiciones de satisfacción y aspiración profesional para establecer un análisis sobre el nivel de satisfacción de los jóvenes universitarios. Se describen los beneficios que aporta el estudio de la satisfacción de aspiraciones profesionales, se justifica la importancia que tiene esta investigación para la ciencia actual y cómo desde las ciencias sociales se les puede dar solución a las situaciones que dificultan el bienestar estudiantil. 


\section{Desarrollo}

En la etapa de la juventud maduran los intereses profesionales, se prioriza la superación y el reconocimiento por la labor que se desempeña. La actividad de estudio adquiere un carácter científico-técnico para aquellos jóvenes que continúan desarrollándose en la Educación Superior, ya que deben asimilar contenidos de diferentes disciplinas que poseen un nivel elevado de abstracción y generalización (DOMÍNGUEZ, 2003). "La elección de la futura profesión constituye un momento esencial y se convierte en el centro psicológico de la nueva situación social del desarrollo" (DOMÍNGUEZ, 2003).

Esto significa que la elección de la profesión puede llegar a ser un verdadero acto de autodeterminación en la medida en que constituya para el joven una aspiración de desarrollo personal. No obstante, la elección profesional puede basarse en otros motivos relacionados con la aprobación social o exclusivamente en la remuneración, estos motivos no excluyen a otros, puesto que constituyen en última instancia motivaciones válidas que regulan el comportamiento de forma efectiva.

Cualesquiera sean los motivos de elección de la profesión, son válidos en la medida en que reflejan una decisión consciente por parte del joven y determinan la adopción de estrategias encaminadas al logro de objetivos. La realización personal del joven en esta importante esfera está condicionada por una adecuada evaluación de sus intereses y capacidades, así como de las principales demandas de la sociedad y, en consecuencia, las posibilidades objetivas de hacer realidad sus aspiraciones.

Por ello, la motivación y la aspiración profesional son elementos significativos que permiten que los jóvenes alcancen exitosamente sus propósitos durante su trayectoria por la Universidad. Sin embargo, ¿se sienten realmente satisfechos los estudiantes universitarios con sus aspiraciones profesionales? ¿cómo la universidad satisface estas aspiraciones?

Los estudios de satisfacción como fenómeno psicológico, han variado mucho a lo largo de los años y han estado muy ligados a las diferentes corrientes y escuelas psicológicas. Las teorías sobre este proceso se basan en los modelos motivacionales, comprendiéndose la motivación como un comportamiento suscitado por necesidades latentes que dirigen al individuo a satisfacerlas o a la obtención de un fin. No obstante, hay que señalar que la satisfacción, comenzó a estudiarse desde las perspectivas de mercado y en su relación con la calidad.

En un análisis sobre la evolución del concepto "satisfacción" en la psicología realizado por Morales Sánchez y Hernández Mendo (2004), se plantea que, en un inicio, desde fines de los 50 del pasado siglo y hasta finales de los 80 , primaba una visión cognitivista sobre el tema, considerándola como una evaluación emocional una vez hecha una compra o uso, como consecuencia del procesamiento de la información relevante. Dicha evaluación puede consistir en una comparación entre las expectativas de los sujetos y el rendimiento que perciben (OLIVER R., 1980), en la comparación social de costes-beneficios (OLIVER; SWAN, 1989a, 1989b), así como en los procesos de atribución que realizan los sujetos (FOLKES; KOLETSKY; GRAHAM, 1987). Ya en los años 90, se comienza a defender el papel activo del fenómeno. Diferentes autores reconocen la aparición de una serie de elementos subjetivos que van acompañados de emociones y estados de ánimo.

Para comprender mejor el comportamiento del cliente, sus intereses y expectativas, se desarrollaron modelos de satisfacción basándose en unidades psicológicas: la Orientación Motivacional, Expectativa Motivacional y el Estado de Satisfacción. La definición de un modelo de satisfacción depende de la esfera motivacional-afectiva que son los impulsores de la persona, así como las unidades psicológicas que se interrelacionan entre sí (HARO; CÓRDOVA; CHONG, 2016).

El Modelo tradicional está enfocado en una imagen que genera expectativas sobre la calidad del producto, la calidad del servicio y el valor del servicio, provocando una satisfacción en el individuo y a su vez una fidelización (HARO; CÓRDOVA; CHONG, 2016). Mientras que, el Modelo personológico se centra en brindar conocimiento sobre los productos o servicios que se ofrecen en una organización, considerando que los clientes poseen un nivel de expectativas o cierta orientación 
hacia el servicio, siendo el grado de expectación directamente proporcional con el grado de decisión al requerir el servicio. Correspondiente a esto la organización debe llenar todas las expectativas y además lograr superarlas con un servicio de calidad y amabilidad. Una vez culminado el servicio se evalúa el grado de satisfacción de los clientes, si la experiencia fue del agrado del cliente, los comentarios serán positivos (HARO; CÓRDOVA; CHONG, 2016).

El modelo básico de motivación está formado por tres variables básicas: estímulo (causa), necesidad (deseo, tensión o inconformidad) y objetivo. Siempre que se produce una necesidad se rompe el equilibrio del organismo y se genera un estado de tensión, insatisfacción, inconformidad y desequilibrio que lleva al individuo a desarrollar un comportamiento o acción capaz de descargar la tensión y liberarlo tanto de la incomodidad como del desequilibrio. Si el comportamiento es eficaz, el individuo satisfacerá la necesidad y por ende descargará la tensión provocada. Satisfecha la necesidad el organismo retorna a su estado de equilibrio y se adaptará al ambiente (ALLEN; FLORES, 1998).

Son varios los autores que han estudiado las teorías motivacionales. Sus aportes se centran en comprender las necesidades que presenta el individuo, ya sean básicas o complejas como la autorrealización personal, superación, etc. Entre los autores se destaca Maslow, el cual brinda una panorámica de las necesidades humanas a partir de la jerarquía piramidal. Según este autor, todos tratamos de satisfacer las necesidades de la base (necesidades fisiológicas), las cuales disminuyen su interés motivador a medida que se han logrado obtener, pasando a satisfacer las de un nivel superior (necesidades de seguridad y protección) y así hasta completar la escalera piramidal (necesidades sociales, necesidades de estimación, necesidades de autorrealización y desarrollo de uno mismo).

Para este autor una necesidad satisfecha no origina ningún comportamiento y sólo las necesidades no satisfechas influyen en el comportamiento y lo encaminan hacia el logro de objetivos individuales. Las necesidades más bajas requieren un ciclo motivacional relativamente rápido, en tanto que las más elevadas necesitan uno mucho más largo. Si alguna de las necesidades más bajas deja de ser satisfecha durante un largo período, se hace imperativo y neutraliza el efecto de las más elevadas. Las energías de un individuo se desvían hacia la lucha por satisfacer una necesidad cuando esta existe (ALLEN; FLORES, 1998).

El autor Herzberg en su Teoría de la motivación-higienecitado por Pérez (2015) brindó una perspectiva interesante sobre el proceso motivacional de las personas desde dos factores: la satisfacción (resultados de los factores de motivación) y la insatisfacción (resultado de los factores de higiene). Considera factores de motivación: el trabajo estimulante, sentimiento de autorrealización, reconocimiento de una labor bien hecha, logro o cumplimiento y la responsabilidad mayor.

Como factores de higiene se encuentran factores económicos, condiciones laborales, seguridad, factores sociales, categoría. Cuando los factores higiénicos se deterioran a un nivel inferior del que los individuos consideran aceptable, entonces se produce insatisfacción. Los factores de higiene afectan directamente las actitudes, principalmente la satisfacción y la insatisfacción, si estos factores han sido satisfechos o llevados a niveles que los individuos consideran aceptables, no habrá insatisfacción, pero tampoco una actitud positiva que se destaque.

Estos modelos contribuyeron a la comprensión del cliente en los estudios de satisfacción en el mercado. Entendiéndose satisfacción como un juicio de valor, que es lo que emite la persona cuando se encuentra satisfecha o no respecto a algo. Esta definición engloba la unidad afectivo-cognitiva de la satisfacción, ya que todo juicio valorativo comprende el proceso cognitivo de pensamiento y análisis, comparación y evaluación, que genera estados afectivos motivacionales, virtud de las propias necesidades, ideales, metas y expectativas del sujeto (GENTO; VIVAS, 2003).

Los estudios realizados desde la visión de mercado (la satisfacción del cliente dependerá del acceso a productos que estarán acorde con las necesidades y expectativas del consumidor) han permitido el desarrollo de investigaciones de la satisfacción desde otras perspectivas tales como la educativa, organizacional, aspectos comerciales de relaciones públicas. 
Para poseer una mejor comprensión sobre el tema central del artículo se hace necesario definir la satisfacción estudiantil, así como los beneficios que aporta al desarrollo del individuo.

Surdez, Sandoval y Lamoyi (2018, p. 12) sostienen que en la educación la satisfacción estudiantil se puede conceptualizar como "el bienestar que experimentan los alumnos por sentir cubiertas sus expectativas académicas como resultado de las actividades que realiza la institución para atender sus necesidades educativas". La satisfacción del estudiante con su experiencia universitaria mejora a partir del conocimiento que posee la institución con las expectativas del nuevo ingreso, la accesibilidad al decano y el nivel de conocimientos y profesionalismos de los profesores (GONZÁLES; PINO-JUSTE; PENADO, 2017). Por tanto, la satisfacción estudiantil es una apreciación favorable que hacen los estudiantes de los resultados y experiencias asociadas con su educación en sus propias necesidades y al logro de sus expectativas.

Además, Caballero, Abello y Palacio (2007), citados por Aguirre Alemán, Sainz Barajas, Chinas Valencia y Carvajal Reyes (2018) establecen una correlación entre las variables burnout, ausentismo escolar, compromiso, autoeficacia, rendimiento académico con la satisfacción. Obteniéndose que bajos niveles de satisfacción, bienestar percibido en relación a la institución y los elementos educacionales inciden en el comportamiento, compromiso y vinculación del estudiante. Por tanto, un estudiante satisfecho con la formación recibida identifica y confirma la calidad de la misma, lo que supone una mayor motivación e implicación, pero a su vez se desarrolla un sentimiento de pertenencia y deseos de intervenir en distintos espacios de participación y actividades académicas (GONZÁLES; PINO-JUSTE; PENADO, 2017).

Según Medrano y Pérez (2010), citado por González, Pino-Juste, y Penado (2017) estudios sostienen que aquellos estudiantes que presentan un alto grado de satisfacción con la Universidad se caracterizan por progresar adecuadamente en sus metas académicas, poseer elevadas creencias acerca de sus capacidades para lograr un buen rendimiento en las tareas, desarrollar expectativas positivas respecto a la vida universitaria constatando la existencia de apoyo social para alcanzar sus objetivos educativos.

Los estudios sobre satisfacción estudiantil en las universidades como indicadores para evaluar la calidad educativa son particularmente importantes, porque la satisfacción estudiantil mejora el rendimiento académico (GARBANZO, 2007), reduce la deserción (OSORIO; PÉREZ, 2010; ALVES; RAPOSO, 2005; CABALLERO; ABELLO; PALACIOS, 2007; HIMMEL, 2002), el cambio de carrera de los inscritos y es un requisito para el éxito en el aprendizaje (SINCLAIRE, 2014). A su vez una comprobada calidad educativa fortalece la imagen y prestigio de la institución (SURDEZ-PÉREZ; SANDOVAL-CARAVEO; LAMOYI-BOCANEGRA, 2018).

Los elementos que tradicionalmente se han visto más vinculados a la satisfacción del estudiante desde la institución han sido: enseñanza (conjunto de acciones, métodos,técnicas, evaluaciones que el docente realiza en el proceso de enseñanza), aspectos organizativos y administrativos (organización del tiempo de docencia, prácticas, estudio independiente; elementos logísticos, recursos, materiales e instrumentales; adecuación de los espacios físicos, bibliotecas, áreas deportivas, cafeterías, comedores) y aspectos relacionales (las relaciones de comunicación con docentes, administrativos y organizaciones dentro de la institución).

Álvarez Botello, Chaparro Salinas y Reyes Pérez (2015) concluyen que las variables que afectan la satisfacción del estudiante son: plan de estudios, capacitación y habilidad para la enseñanza de los docentes, métodos de enseñanza y evaluación, nivel de autorrealización del estudiante, servicios de apoyo, servicios administrativos, ambiente propicio e infraestructura.

Muchos de estos estudios tienen como objetivo fundamental estudiar la satisfacción con el afán de validar instrumentos sobre alguna base teórica. En el análisis de estos instrumentos ha quedado demostrado que el fenómeno de la satisfacción es multidimensional. El principal sesgo de gran parte de las investigaciones consultadas es que no exploran aquellos elementos que aporta el usuario al fenómeno. En buena parte de los casos no se exploran las expectativas, necesidades 
y objetivos de los sujetos al establecer la relación, y menos, en el caso de los estudiantes universitarios, los elementos propios que le permitirán autosatisfacerse en la relación con el servicio educativo, en este caso, motivación, disponibilidad de tiempo y condiciones para el estudio fuera de la institución, entre otros.

Un ejemplo que sí explora algunos elementos subjetivos del estudiante es el caso de la investigación realizada por Álvarez-Vaz y Vernazza (2017) que, con el criterio del estudiante como cliente y la Universidad como un servicio de educación terciaria, buscan establecer cómo se construye el concepto de satisfacción estudiantil a través de la aplicación de un cuestionario formado por conjuntos de preguntas que conforman el modelo ECSI (European Costumer Satisfaction Index). En este caso el análisis de la información recogida fue cuantitativo mediante el Análisis de Clases de Latentes y el Análisis de Clusters. El aporte de este estudio está en el empleo de los métodos estadísticos mencionados para identificar grupos de los resultados obtenidos y su recíproca comprobación.

Los estudios que relacionan la satisfacción estudiantil y las aspiraciones profesionales son muy escasos. En Cuba se han llevado a cabo investigaciones sobre las aspiraciones de jóvenes universitarios desde una categoría más amplia, proyectos futuros. Los estudios tutorados por la Dra. Laura Domínguez: Ortet Gonzáles (2011); Pérez Rodríguez \& Ramos Díaz (2012); Alfonso Bezares (2013); Morales Gutiérrez (2013); Zaneti Díaz (2016) definen los proyectos futuros como una categoría psicológica y formación motivacional compleja de la personalidad. En ellos se entienden las aspiraciones como expresión de los ideales.

Ortet Gonzáles (2011) sostiene que, en cuanto al desarrollo de los proyectos futuros, en el contenido de los mismos se refleja la aspiración de los estudiantes a graduarse y poder superarse en el ámbito profesional, obtener éxito en esta esfera les hace sentir realización personal y profesional. La autora afirma que los objetivos, aspiraciones y metas que integran los principales proyectos futuros de los jóvenes se asocian a esferas tales como, la profesión, el estudio, el trabajo, la familia, la realización personal, el empleo del tiempo libre y la búsqueda de caminos que les permitan satisfacer sus necesidades materiales.

Por otra parte, para Ayala García (2014, p. 6), las aspiraciones individuales tienen su origen en tres grupos de factores: los factores individuales, los familiares y los del vecindario o la comunidad.

Dentro de los factores individuales se encuentran características relacionadas con la inteligencia, los logros individuales, la depresión y el estrés psicológico, [...] dentro de los factores familiares se encuentran la clase social de origen, el nivel socioeconómico familiar, la cantidad de recursos con los que se cuente, y el estímulo que se recibe por parte de los padres, [...] por último, los factores relacionados con el vecindario o la comunidad corresponden al mundo cognitivo de las personas, de las experiencias, vidas, logros e ideales de aquellas personas pertenecientes a su entorno cercano, y se pueden ver afectadas por la percepción de movilidad social en sociedades altamente desiguales, las experiencias vividas por personas cercanas y por la existencia o conocimiento de mecanismos que permitan salir de la pobreza.

Entrialgo, Fernández y Vázquez (2000) por su parte, plantean que las aspiraciones presentan dos dimensiones. De un lado, las aspiraciones pueden ser intrínsecas o extrínsecas. Las primeras ponen el acento en el trabajo mismo como fuente de satisfacción esperada; las segundas sitúan la satisfacción en los resultados del trabajo. De otro lado, las aspiraciones pueden ser individuales u organizativas (internas o externas). Las segundas, a diferencia de las primeras, requieren el concurso del equipo, es decir, se ven satisfechas potencialmente en un ámbito que requiere la coordinación de esfuerzos individuales distintos. Las primeras, en cambio, se refieren al individuo como fuente última de satisfacción de las aspiraciones. Se puede observar, claramente, una analogía entre ambos tipos de aspiraciones.

Entonces, al hablar de aspiraciones estamos reflejando una construcción basada en los ideales, necesidades, objetivos, metas y motivos que se han asimilado y reforzado a lo largo de los años. Es 
decir, constituyen estructuras motivacionales que alcanzan estabilidad en su organización en la etapa de la juventud, integrándose en la proyección futura y concepción del mundo, la cosmovisión de los sujetos. Se puede decir que se han constituido en la personalidad del individuo una vez han alcanzado la integración cognitivo-afectivo-motivacional, evidenciándose en comportamientos coherentes con ellas, siendo mediadoras de las expectativas de los sujetos.

En el caso particular de las aspiraciones profesionales se hace referencia a aquellas que se encuentran directamente relacionadas con la preparación y vida profesional. Entonces, vamos a entender como aspiraciones profesionales aquello que el sujeto expresa que pretende lograr, hacer, alcanzaren la esfera formativo-profesional. No solo el simple deseo, sino que está acompañado por un sistema de objetivos, motivos y metas y la disposición comportamental.

En el contexto cubano, los resultados de la IV Encuesta Nacional de Juventud (CESJ; ONEI, 2012) realizada por el Centro de Estudios Sobre la Juventud, evidencian que el $69 \%$ de los encuestados aspira a realizar estudios universitarios, siendo más las mujeres $(74,4 \%)$ que los hombres $(63,3 \%)$ con dicha aspiración. Muestran como principales razones de superación: ampliar los conocimientos y ser una persona preparada en la vida. Sin embargo, en el caso de los jóvenes estudiantes, se observan también otros motivos de carácter extrínseco, como la posibilidad de satisfacer sus necesidades económicas y la complacencia de los padres. Estos motivos se reiteran también como la razón fundamental para estudiar en la II y III encuesta nacional.

Según Estévez (2014) en Modificaciones educacionales en Cuba entre el 2010 y el 2014. Repercusión en los adolescentes y jóvenes, los motivos para la continuidad de estudios están relacionados con el interés por la profesión, así como la posibilidad de un salario mayor y un estatus socio-económico más elevado, también se replican en los resultados de otras investigaciones cubanas sobre el tema, por lo que podemos afirmar que estas razones caracterizan, al menos en su generalidad, a la juventud cubana.

La relación del estudiante cubano con sus instituciones educativas, según los resultados de la IV ENJ plantea que "la gran mayoría de los jóvenes consideran que la escuela cubana le brinda suficiente preparación para asumir determinadas situaciones en la vida, como la vida laboral y el trabajo en colectivo" (CESJ; ONEI, 2012). Aunque son alentadores estos resultados en cuanto a la existencia de un clima favorable en la relación del estudiante con su institución educativa, también se observa la existencia de insatisfacciones relacionadas a insuficiencias en la formación, según la perspectiva de los estudiantes. En relación con esto último, también son coherentes los resultados de Estévez (2014, p. 10) que plantea que "para los integrantes de nivel superior y medio superior, los niveles de insatisfacción con la orientación profesional son relativamente altos".

Exactamente por estos resultados se hace necesario estudiar la satisfacción de aspiraciones profesionales. Investigaciones revelan que cada año hay estudiantes que deciden dejar sus carreras, ya sea por necesidad económica, porque sus expectativas no se corresponden a lo que reciben en la Universidad o por problemas personales que dificultan la continuidad de estudios.

La educación de las nuevas generaciones es una prioridad en el país, por ello su perfeccionamiento exige reconocer aquellas fisuras del sistema educativo que puedan estar deteriorando el proceso de aprendizaje estudiantil. Teniendo en cuenta esto, la satisfacción es una variable que, como bien se decía antes, influye en las emociones positivas que sienten los estudiantes hacia la institución, además refuerza el sentido de pertenencia y la identidad organizacional. ¿Sienten eso nuestros estudiantes universitarios cubanos? Se podría decir que sí, las instituciones universitarias del país otorgan elementos característicos de la organización para garantizar la participación estudiantil. Sin embargo, algunas instituciones prefieren entregar elementos identitarios solo a aquellos estudiantes que participan en actividades merecedoras de ese reconocimiento, excluyendo a aquellos que, a pesar de sentirse motivados hacia su formación, no se interesan hacia otras actividades que organiza la Universidad.

Además, el ambiente en el que se desarrolla el estudiante, las expectativas que genera dentro de la institución, influyen en la relación estudiante-organización. En nuestro país, el recrudecimiento 
del bloqueo ha dificultado seriamente que las condiciones de los centros educativos sean óptimas, así como que posean los recursos necesarios para favorecer la asimilación del conocimiento en los estudiantes. Actualmente, se cuentan con recursos escasos y desactualizados, incluso si se deterioran a tal punto que son inutilizables, debe intervenir la creatividad del profesor para que los jóvenes estudiantes logren apropiarse de la información que este quiere trasmitir.

La sociedad cambia, los jóvenes se sienten cada vez más atraídos hacia la independencia y la obtención de ganancias económicas. En el siglo pasado la juventud priorizaba los estudios universitarios, lo cual garantizaba un futuro profesional próspero. En estos tiempos los jóvenes inician sus estudios por diferentes motivos: complacer a la familia, obtener un buen trabajo después de graduado, ser un excelente profesional, viajar, etc. Sin embargo, una parte significativa de los estudiantes que inician sus estudios no se gradúan. Algunos se desapegan totalmente de la carrera y modifican sus aspiraciones profesionales, pudiendo concebir el trabajo que realizan como un medio más viable para alcanzar sus objetivos. Otros, simplemente, cambian de carrera porque no se sienten identificados con la que iniciaron. Además, cada vez es mayor la cantidad de jóvenes que se mantienen trabajando y estudiando a la par, por lo que deciden continuar su formación académica incorporándose a los cursos por encuentros.

El estudio de la satisfacción de aspiraciones profesionales es un problema actual porque no se profundiza en la satisfacción estudiantil y se utiliza a favor del desarrollo del aprendizaje. Dominar únicamente el resultado académico o las asignaturas al que el estudiante le atraen más, no les permite saber a los profesores y directivos si estos están cumpliendo sus aspiraciones dentro del centro educativo. Es probable que los docentes no tengan conocimiento de los proyectos futuros de sus estudiantes, ni de los elementos psicológicos que intervienen en el proceso de satisfacción como: personalidad, identidad, intereses, motivaciones, expectativas, así como los elementos externos: cultura, contexto, relaciones sociales, programa educativo, condiciones de estudio.

De ser conscientes de esos procesos no se limitarían a dar las clases correspondientes en el programa educativo, sino que indagarían cómo se siente el estudiante, qué expectativas tiene del curso escolar y de las asignaturas que recibe, qué aspiraciones posee, qué correspondencia existe entre lo que estudia y lo que aspira. Teniendo estas informaciones conocemos al estudiante y elaboramos programas que se correspondan con sus necesidades e intereses.

Las ciencias sociales pueden investigar el proceso de satisfacción de aspiraciones profesionales en jóvenes universitarios debido a que se obtiene una visión actualizada y contextualizada sobre el proceso a investigar y se identifican los elementos que inciden en el fenómeno. Realizar este estudio, beneficiaría la creación, perfeccionamiento e implementación de políticas y programas que permitan elevar la satisfacción percibida por los jóvenes universitarios cubanos, en su tránsito por la Universidad.

Desde esta investigación se pueden identificar los elementos que inciden en el proceso de satisfacción e intervenir para que aumente la calidad del aprendizaje en los universitarios. Además, brindar conciencia de lo que se está descuidando y dan pistas de cómo actuar para mejorar, ya sea aportando herramientas sociopsicológicas para interactuar con los jóvenes, desarrollando programas innovadores para potenciar la motivación o realizando espacios grupales. No obstante, las acciones a llevar a cabo le corresponden a la institución educativa, así que, si existe insatisfacción hacia el ambiente en el que estudian los jóvenes, las clases que reciben, las actividades escolares, el sistema comunicativo del centro escolar, etc., los investigadores sociales los detectan e informan a la institución educativa para que participen en la intervención. Sería una colaboración conjunta, porque es posible realizar transformaciones significativas si la Universidad así lo desea.

Satisfacer a los estudiantes universitarios proporcionaría, además de múltiples ventajas para los jóvenes, mayor reconocimiento a la Universidad tanto nacional como internacional, su prestigio se elevaría y serían más visibles los resultados que han llevado a cabo sus estudiantes y docentes. Aumentaría la atracción de los jóvenes hacia ese espacio educativo, así como la lealtad 
y transmisión de la experiencia positiva vivida a otras personas. Incluso, el interés de los jóvenes hacia la docencia podría incrementarse, siendo probable que deseen motivar a otros a alcanzar su meta desde sus vivencias positivas.

La calidad del proceso educativo universitario cubano es admirado en países latinoamericanos. Se reconoce que el país cuenta con profesionales capacitados para garantizar que las nuevas generaciones posean una amalgama amplia de conocimientos. Sin embargo, siempre la educación se puede perfeccionar y qué mejor manera que conociendo si los estudiantes se encuentran satisfechos o no con los servicios que ofrece la universidad para alcanzar sus proyectos futuros.

$\mathrm{Al}$ fin y al cabo, por los jóvenes es por quienes se apuesta. Démosles la oportunidad de que se tengan en cuenta sus motivaciones. Medir la satisfacción de los estudiantes se fundamenta en el hecho de que son ellos el eje nuclear y la garantía de la existencia y mantenimiento de las organizaciones educativas. Los estudiantes como destinatarios de la educación son los que mejor pueden valorarla y, aunque puedan presentar una visión parcial y subjetiva, su opinión proporciona un referente que debe tomarse en cuenta. Llevar a cabo este proceso sería para realizar acciones que promuevan la mejora y la innovación en las instituciones educativas (GONZÁLEZ; PINO-JUSTE; PENADO, 2017).

Actualmente no se puede concluir si los jóvenes se sienten o no satisfechos con sus aspiraciones profesionales en la universidad, ni cómo esta satisface esas aspiraciones porque este estudio está en fase de aplicación por lo que aún no se ha podido obtener resultados. Sin embargo, es posible que en diferentes aspectos en los que nos proponemos investigar los universitarios se encuentren satisfechos con su formación educativa: sintiéndose a gusto con la carrera que estudian, correspondiéndose sus expectativas en alguna medida con la realidad y desarrollando aspiraciones que la universidad podría satisfacer.

Metodológicamente el estudio se plantea como Objetivo General diagnosticar la satisfacción de las aspiraciones profesionales de los estudiantes universitarios cubanos en su tránsito por la Educación Superior en el curso 2020-2021. Para lograrlo se establecieron los objetivos específicos de identificar las aspiraciones profesionales de los estudiantes universitarios cubanos en el curso 2020-2021, constatar el nivel de satisfacción de los jóvenes universitarios cubanos respecto a sus aspiraciones profesionales y determinar los factores de la relación estudiante-Universidad que influyen en la satisfacción de los jóvenes universitarios cubanos respecto a sus aspiraciones profesionales.

\section{Conclusiones}

El estudio de la satisfacción estudiantil aporta ventajas como la lealtad, la permanencia universitaria, bienestar psicológico, identificación, compromiso y mejores relaciones estudiante-estudiante y estudiante-profesor. La mayoría de los estudios sobre satisfacción han estado dirigidos a la evaluación de la calidad educativa para mantener el prestigio de la Universidad y la obtención de ganancias económicas desatendiéndose elementos como la motivación y las aspiraciones de los estudiantes.

En Cuba las aspiraciones profesionales se estudian desde una categoría más amplia: los proyectos futuros. Este proceso moviliza al sujeto a elaborarse metas y proyectos a alcanzar dentro del contexto profesional.

La satisfacción de aspiraciones profesionales es un problema para la ciencia actual porque sus resultados aportarían recursos para desarrollar programas innovadores y creativos que favorecerían la motivación y mejoraría la calidad del proceso enseñanza-aprendizaje de los universitarios cubanos.

Existen elementos externos e internos que influyen en la satisfacción de las aspiraciones profesionales: motivación, intereses, identidad, personalidad, así como ambiente, programa educativo, relaciones escolares, condiciones de estudio. Conocer estos elementos le brinda al docente herramientas sobre las que trabajar para establecer un mejor vínculo profesor-estudiante. 


\section{Referencias}

AGUiRRE AlEMÁN, María G.; SAINZ BARAJAS, María T.; CHINAS VALENCIA, Juan J.; CARVAJAL REYES, Arantxa. Satisfacción estudiantil en Facultad de Contaduría y Administración. Universidad Veracruzana, Coatzacoalcos. Revista Internacional la Nueva Gestión Organizacional, México, n. 9, p. 61-79, 2018. Disponible en: https:// www.researchgate.net/profile/Flor-Gomez-

Reyes/publication/330358244_Negocios_inclusivos_un_analisis_en_la_localidad_de_Kennedy_en_BogotaColombia/ links/5c3c1656458515a4c72481d6/Negociosinclusivos-

un-analisis-en-la-localidad-de-Kennedy-en-BogotaColombia.pdf. Acceso en: 17 dic. 2019.

ALFONSO BEZARES, Cindia T. Proyectos profesionales y valores declarados asociados al desempeño profesional en estudiantes del la Licenciatura en Artes Visuales del Instituto Superior de Arte. 2013. Trabajo de Diploma - Facultad de Psicología, Universidad de La Habana, La Habana, 2013.

ALLEN, Ana Marilin; FLORES, Grettchen. Análisis de la motivación del recurso humano. Comparación entre los servicios de cirugía del hospital Dr. Calderón Guardia y patologia forense del Organismo de Investigación Judicial. Medicina Legal, Costa Rica, v. 15, n. 1-2, p. 11-17, 1998. Disponible en: https://www.scielo.sa.cr/scielo.php?pid=S1409-00151998000200006\&script=sci_abstract\&tlng=es. Acceso en: 15 nov. 2019.

ALVES, Helena; RAPOSO, Mario. La medición de la satisfacción en la enseñanza universitaria: el ejemplo de la Universidad de da Beira Interior. International Review

on Public an Nonprofit Marketing, [s. l.], v. 1, n. 1, p. 73-88, 2005. Disponible en: https://www.researchgate.net/publication/225663483_La_medicion_de_la_satisfaccion_en_la_ensenanza_universitaria_El_ejemplo_de_la_Universidade_da_Beira_Interior. Acceso en: 14 sept. 2020.

ÁlVAREZ BOTELLO, Julio; CHAPARRO SALINAS, Eva Martha; REYES PÉREZ, Diana Elena. Estudio de la satisfacción de los estudiantes con los sercicios educativos brindaddos por Instituciones de Educación Superior del Valle de Toluca. Revista Iberoamericana sobre Calidad, Eficacia y Cambio en Educación, México, v. 13, n. 2, p. 5-26, 2015. Disponible en: https://revistas.uam.es/index.php/reice/article/view/2788. Acceso en: 26 nov. 2019.

ÁLVREZ-VAZ, Ramón; VERNAZZA, Elena. Satisfacción Estudiantil: análisis desde una perspectiva multivariante. Serie Documentos de Trabajo. Instituto de Estadística, Facultad de Ciencias Económicas y de Administración, Universidad de la República, Montevideo, oct. 2017. documento. Disponible en: https://www.researchgate.net/publication/321478537_SATISFACCION_ESTUDIANTIL_ANALISIS_DESDE_UNA_PERSPECTIVA_MULTIVARIANTE. Acceso en: 15 dic. 2019.

AYALA GARCÍA, Jhorland. Documentos de trabajo sobre economía regional: Aspiraciones económicas, conflictos y trampas de pobreza en Colombia. Cartagena: Banco de la República: Centro de Estudios Económicos Regionales, 2014. v. 212. Disponible en: https://www.banrep.gov.co/sites/default/files/publicaciones/archivos/dtser_212.pdf. Acceso en: 17 feb. 2020.

CABALLERO, Carmen Cecilia; ABELLO, Raymundo; PALACIOS, Jorge. Relación del burnout y el rendimiento académico con la satisfacción frente a los estudios en estudiantes universitarios. Avances en Psicología Latinoamericana, Colombia, v. 25, n. 2, p. 98-111, 2007. Disponible en: https://www.redalyc.org/articulo.oa?id=79925207. Acceso en: 17 feb. 2020.

CENTRO DE ESTUDIOS SOBRE LA JUVENTUD (CESJ); OFICINA NACIONAL DE ESTADÍSTICA E INFORMACIÓN (ONEI). Cuarta Encuesta Nacional de Juventud. Informe de Investigación. La Habana: CESJ: ONEI, 2012.

DOMÍNGUEZ GARCÍA, Laura. Psicología del Desarrollo: Adolescencia y Juventud. La Habana: Félix Varela, 2003.

DOMÍNGUEZ GARCÍA, Laura; IBARRA MUSTELIER, Lourdes. Selección de lecturas. Psicología del Desarrollo: adolescencia y juventud. La Habana: Felix Varela, 2003.

ESTÉVEZ, Keyla Rosa. Modificaciones educacionales en Cuba entre el 2010 y el 2014. Repercusión en los adolescentes y jóvenes. Documentos de trabajo. La Habana: Centro de Estudios Sobre la Juventud, 2014.

FOLKES, Valerie S.; KOLETSKY, Susan; GRAHAM, John L. Un estudio de campo de inferencias causales y reacción del consumidor: la vista desde el aeropuerto. Journal of Consumer Research, [s. l.], v. 13, n. 4, p. 534-539, 1987. Disponible en: https://psycnet.apa.org/record/1987-26860-001. Acceso en: 5 sept. 2019.

GARBANZO VARGAS, Guiselle María. Factores asociados al rendimiento académico en estudiantes universitarios, una reflexión desde la calidad de la educación superior pública. Revista Educación, Costa Rica, v. 31, n. 1, p. 43-63, 2007. Disponible en: https://revistas.ucr.ac.cr/index.php/educacion/article/view/1252. Acceso en: 5 sept. 2019.

GENTO PALACIOS, Samuel; VIVAS GARCÍA, Mireya. El SEUE: un instrumento para conocer la satisfacción de los estudiantes universitarios con su educación. Acción Pedagógica, Venezuela, v. 12, n. 2, p. 16-27, 2003. Disponible en: https://dialnet.unirioja.es/servlet/articulo?codigo=2972060. Acceso en: 5 marzo 2019. 
GONZÁLES, Margarita; PINO-JUSTE, Margarita; PENADO, María. Estudio de la satisfaccion percibida por los estudiantes de la UNED con su vida universitaria. Revista Iberoamericana de Educación a Distancia, Madrid, v. 20, n. 1, p. 243-260, 2017. Disponible en: http://revistas.uned.es/index.php/ried/article/view/16377/21967. Acceso en: 9 oct 2019.

HARO CARRILLO, Fernando Andrés; CORDOVA FLORES, Nelson Claudio; CHONG QUI ESTEVES, Tomás Bernardo. Modelos de Satisfacción: Fundamentación Teórica y Criterios de Aplicación. INNOVA Research Journal, Ecuador, v. 1, n. 10, p. 54-62, 2016. Disponible en: https://revistas.uide.edu.ec/index.php/innova/article/view/64. Acceso en: 17 dic. 2019.

HIMMEL, Erika. Modelos de análisis de la deserción estudiantil en la educación superior. Revista Calidad en la Educación, Santiago de Chile, v. 17, p. 91-108, 2002. Disponible en: https://www.calidadenlaeducacion.cl/index.php/rce/ article/view/409. Acceso en: 5 sept. 2019.

MORALES GUTIÉRREZ, Néstor R. Proyectos profesionales y valores en estudiantes de quinto año de la carrera de Medicina. 2013. Trabajo de Diploma - Departamento de Psicología, Facultad de Ciencias Médicas de Matanzas Dr. Juan Guiteras Gener, Matanzas, 2013.

MORALES SÁNCHEZ, Verónica; HERNÁNDEZ MENDO, Antonio. Calidad y satisfacción en los servicios: conceptualización. Revista Digital, Buenos Aires, n.73, 2004. Disponible en: https://www.efdeportes.com/efd73/calidad. htm\#: :text=Desde\%20esta\%20perspectiva\%2C\%20la\%20calidad,o\%20utilizan\%20estos\%20bienes\%20de. Acceso en: 21 nov. 2018.

OLIVER, Richard L. A cognitive model of the antecedents and consequences of satisfaction decision. Journal of Marketing Research, EUA, v. 17, p. 460-469, 1980. Disponible en: https://journals.sagepub.com/doi/abs/10.1177/002224378001700405. Acceso en: 6 nov. 2019.

OLIVER, Richard L.; SWAN, John E. Consumer perceptions of interpersonal equity and satisfaction in transactions: a field survey approach. Journal of Marketing, EUA, v. 53, n. 2, p. 21-35, 1989a. Disponible en: https://www.jstor.org/ stable/1251411. Acceso en: 6 nov. 2019.

OLIVER, Richard L.; SWAN, John E. Equity and disconfirmation perceptions as intluences on merchant and product satisfaction. Journal of Consumer Research. Journal of Consumer Research, EUA, v. 16, p. 372-383, 1989b. Disponible en: https://academic.oup.com/jcr/article-abstract/16/3/372/1818813. Acceso en: 6 nov. 2019.

ORTET GONZÁLES, Beatriz. Proyecto de vida y valores en estudiantes de la Licenciatura en Biología de la Universidad de La Habana. 2011. Tesis (Licenciatura en Psicología) - Facultad de Psicología, Universidad de la Habana, La Habana, 2011.

OSORIO, Jocelyn Viviana; MÓNICA PÉREZ, Karla. El nivel de satisfacción escolar y su relación con la orientación vocacional en alumnos de psicología educativa. 2010. Tesis (Licenciatura en Pedagogía) - Universidad Pedagógica Nacional, México, 2010.

PÉREZ, Alejandro. La Teoría de la Motivación-Higiene de Herzberg. In: CEOLEVEL. [S. l.], 22 jun. 2015. Disponible en: http://www.ceolevel.com/herzberg. Acceso en: 14 sept. 2020.

PÉREZ RODRÍGUEZ, Lilian; RAMOS DÍAZ, Raisa. Proyectos profesionales y valores asociados al desempeño de la profesión en estudiantes de la Licenciatura en Economía de La Universidad de la Habana. 2012. Trabajo de DiplomaFacultad de Psicología, Universidad de La Habana, La Habana, 2012.

SINCLAIRE, Jollean. An empirical investigation of student satisfaction with college courses. Research in Higher Education Journal, [s. l.], v. 1, n. 21, 2014. Disponible en: www.aabri.com>manuscripts. Acceso en: 6 nov. 2019.

SURDEZ-PÉREZ, Edith G.; SANDOVAL-CARAVEO, María del Carmen; LAMOYI-BOCANEGRA, Clara L. Satisfacción estudiantil en la valoración de la calidad educativa universitaria. Educación y Educadores, Colombia, v. 21, n. 1, p. 9-26, 2018. Disponible en: www.redalyc.org>jatsRepo>html. Acceso en: 6 nov. 2019.

VÁSQUEZ ORDÁS, Camilo José; FERNÁNDEZ SÁNCHEZ, Esteban; ENTRIALGO SUÁREZ, Montserrat. Una taxonomía de las aspiraciones laborales del propietario/directivo de PYMEs. Documentos de trabajo: Facultad de Ciencias Económicas y Empresariales, Espanha, n. 208, 2000. Disponible en: https://dialnet.unirioja.es/servlet/articulo?codigo=1252840. Acceso en: 5 sept. 2019.

ZANETI DÍAZ, Patricia. Características de los proyectos futuros de un grupo de jóvenes universitarios del Curso Regular Diurno que estudian y trabajan. 2016. Tesis (Licenciatura en Psicología) - Facultad de Psicología, Universidad de La Habana, La Habana, 2016.

Data de submissão: 29/01/2021

Data de aceite: 05/02/2021 
